\title{
Cartografía analógica y digital para la delimitación regional y el análisis temático: aplicación a la cuenca del río Luján (Argentina) ${ }^{1}$
}

\author{
Gustavo D. Buzai², Sonia L. Lanzelotti³, Luis Fernando Paso Viola ${ }^{4}$ \\ y Noelia Principi ${ }^{5}$
}

\begin{abstract}
RESUMEN
El paso inicial del proyecto "Análisis espacial y evaluación de zonas de potenciales conflictos ambientales, productivos y patrimoniales ante la expansión urbana en la cuenca del río Luján (Provincia de Buenos Aires, Argentina)" consistió en realizar la actualización de la delimitación del área de estudio. Para llevar a cabo esta tarea fue trazada una secuencia teórico-metodológica que implicó el análisis de cartografía histórica, confección de mapas mediante cartografía asistida por computador (CAC), definición de límites mediante el procesamiento digital de imágenes satelitales (PDI) utilizando modelos digitales de elevación y realización de la base de datos geográfica a través de Sistemas de Información Geográfica (SIG) vinculados con los globos terráqueos virtuales. El presente trabajo presenta aspectos teórico-metodológicos que permiten ejemplificar la secuencia con detalle en la alta cuenca, tanto en aspectos cartográficos como en imágenes logradas en el trabajo de campo y uso de GPS. El total de la tarea presenta claros aspectos de la vinculación entre procedimientos tradicionales y las actuales tecnologías de la información geográfica (TIG) utilizadas en la construcción regional y la representación temática de los resultados.
\end{abstract}

Palabras clave: Mapa Topográfico, Mapa Temático, Cartografía Digital, Sistemas de Información Geográfica, Tecnologías de la Información Geográfica.

\begin{abstract}
The initial step of the research project "Spatial analysis and evaluation of areas of environmental, productive and patrimonial potential conflicts before the urban expansion in the Luján river basin (Province of Buenos Aires, Argentina)" consisted in updating the delimitation of the study area. In order to carry out this task a theoretical-methodological sequence was drawn up, which involved the analysis of historical cartography, mapping using computer-aided cartography (CAC), definition of boundaries using digital image processing (DIP) through digital elevation models (DEM) and realization of the geographic database through Geographical Information Systems (GIS) linked to Virtual Earth and Digital Globes. The present work presents theoretical and methodological aspects that allow to exemplify the sequence with detail in the high basin area, as much in cartographic aspects as in images obtained in the field work with GPS. The total of the task presents clear aspects of the link between traditional procedures and the current Geographical Information Technologies (GIT) used in the regional construction and thematic representation of the results.
\end{abstract}

Keywords: Topographic Map, Thematic Map, Digital Cartography, Geographical Information Systems, Geographic Information Technologies.

El presente trabajo fue realizado en el marco del proyecto PICT 2014-1388 de la Agencia Nacional de Promoción Científica y Tecnológica (ANPCyT) de la República Argentina, financiado por el Fondo para la Investigación Científica y Tecnológica (FONCyT) y radicado en el Instituto de Investigaciones Geográficas (INIGEO) de la Universidad Nacional de Luján. Artículo recibido el 14 de agosto de 2017, aceptado el 9 de enero de 2018 y corregido el 30 de enero de 2018.

Instituto de Investigaciones Geográficas, Universidad Nacional de Luján y Consejo Nacional de Investigaciones Científicas y Técnicas (Argentina). E-mail: buzai@unlu.edu.ar

3 Instituto de Investigaciones Geográficas, Universidad Nacional de Luján y Consejo Nacional de Investigaciones Científicas y Técnicas (Argentina). E-mail: sonia.lanzelotti@conicet.gov.ar

Instituto de Investigaciones Geográficas, Universidad Nacional de Luján (Argentina). E-mail: fpasoviola@yahoo.com.ar

Instituto de Investigaciones Geográficas, Universidad Nacional de Luján (Argentina).E-mail:nprincipi@unlu.edu.ar 
El uso de cartografía, ante la consideración del mapa como un sistema de información, brinda la posibilidad de contar con una base teórico-metodológica que se presenta en diferentes instancias durante la totalidad del proceso de investigación que inicia con la necesidad de definir el área de estudio y culmina con una síntesis de las distribuciones espaciales de los resultados obtenidos.

Esta perspectiva considera el formato papel (Dacey, 1970) y el formato digital ligado a la informática (González Leiva, 1984), de esta forma es posible apoyar de manera continua toda investigación en la que la dimensión espacial resulta ser central.

La definición del área de estudio implica poner límites en el espacio geográfico, lo cual constituye una de las principales tareas de la Geografía como ciencia apoyada en una perspectiva racionalista.

La región se construye utilizando la materialidad empírica encontrada en la superficie terrestre. Sin embargo, cuando se apela al concepto de cuenca hidrográfica la construcción regional se simplifica a partir de trazar límites apoyados en los puntos más elevados de las divisorias de agua. Inicialmente en el siglo XVIII el geógrafo francés Philippe Buache (1700-1773) había considerado que la división del espacio geográfico por cuencas hidrográficas brindaba una solución objetiva a la delimitación regional.

La investigación "Análisis espacial y evaluación de zonas de potenciales conflictos ambientales, productivos y patrimoniales ante la expansión urbana en la cuenca del río Luján (Provincia de Buenos Aires, Argentina)" tuvo dos objetivos específicos: (1) realizar la actualización de la delimitación del área de estudio y (2) analizar diferentes problemáticas socioespaciales en su interior.

El primer objetivo surge a partir de detectar la inexistencia de una definición espacial clara para los límites de la cuenca del río Luján estando vigente la aproximación realizada con finalidad didáctica en Buzai (2002). Por lo tanto, fueron combinados secuencialmente diversos recursos metodológicos y técnicos con la finalidad de realizar una nueva definición espacial que contemple un nivel de detalle apropiado para una herramienta de planificación. En el presente trabajo se verá cómo se cumple dicho objetivo combinando el uso de cartografía topográfica, cartografía digital, trabajo de campo, imágenes satelitales con el modelo digital de elevación (DEM), ajustes específicos en los límites de la cuenca inferior y superior, y su incorporación al Sistema de Información Geográfica raster y vectorial con su vinculación al globo terráqueo virtual (GTV).

El segundo objetivo, una vez logrado el primero, se basa en el análisis espacial cuantitativo con SIG aplicado al estudio de problemáticas socioespaciales apoyadas principalmente en los conceptos de distribución y asociación espacial. En el presente trabajo se incluyen, como ejemplos, dos aplicaciones: una en formato vectorial basada en procedimientos de análisis multivariado para la construcción de clasificaciones espaciales con variables sociodemográficas y una en formato raster basada en la aplicación de técnicas de evaluación multicriterio para la detección de zonas de potenciales conflictos ante la evolución espacial de los usos del suelo. La cartografía temática se presenta al final de cada proceso aplicativo en su capacidad de síntesis geográfica.

El ambiente natural presenta a la cuenca del río Luján como una subcuenca de la gran cuenca del Plata. Su topografía general es suave con máximas alturas en el Oeste con valores de cota 
superiores a los 50 metros que descienden de forma continua durante una extensión de más de 100 kilómetros en sentido noreste hasta alcanzar el nivel del mar.

El curso principal es el río Luján que realiza su recorrido por una planicie con pequeñas ondulaciones. En algunos tramos se encuentra canalizado y es recto (Figura $N^{\circ} 1$ ) y en otros muestra un recorrido sinuoso de meandros (Figura $\mathrm{N}^{\circ} 2$ ).

Figura $N^{\circ} 1$

Río Luján canalizado en la ciudad de Luján

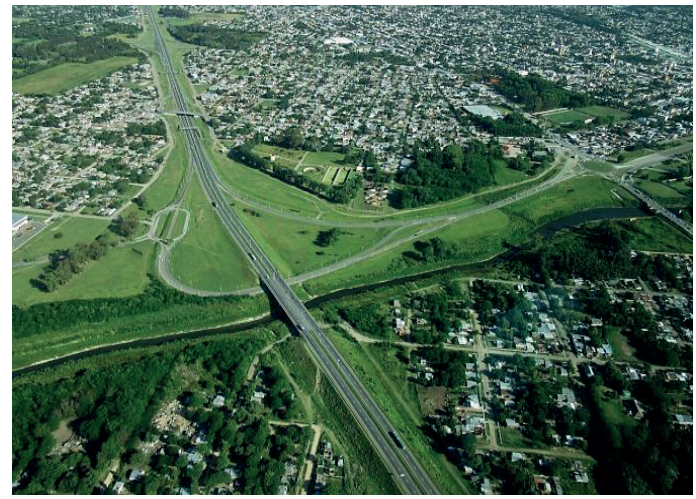

Fuente: Archivo fotográfico personal de Gustavo Buzai.
Figura $\mathrm{N}^{\circ} 2$

Río Luján con meandros en el municipio de Pilar

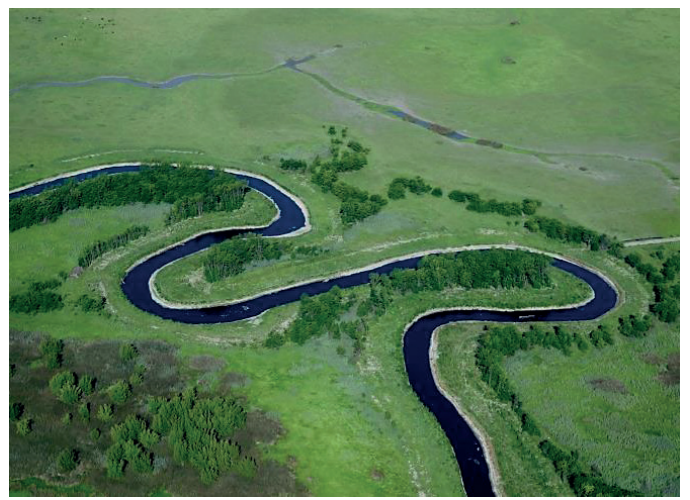

Fuente: Archivo fotográfico personal de Noelia Principi.

En síntesis, el presente trabajo detalla las características del proyecto de investigación centrando su atención en aquellos aspectos metodológicos que se apoyan la representación cartográfica, tanto como base para lograr una nueva definición espacial del área de estudio como para generar representaciones temáticas de los resultados. Por lo tanto, el recorrido teórico-metodológico se centra en las dos grandes tendencias de la Cartografía General: la Topográfica y la Temática (González Leiva, 1986). La primera de ellas en la consideración de la materialidad concreta de los elementos sobre la superficie terrestre que resulta de utilidad para orientar toda delimitación espacial y la segunda utilizada para representar espacialmente los temas analizados y los resultados obtenidos a través de la aplicación de procedimientos de análisis espacial.

\section{El proyecto de investigación}

La investigación se focalizó en el estudio de las problemáticas surgidas ante la dinámica de expansión urbana en la cuenca del río Luján (Buzai et al., 2015). Se analizó el impacto de la evolución espacial de la aglomeración sobre ambientes rurales y de conservación para los partidos de la cuenca, sobre los sitios de interés patrimonial (arqueológicos, paleontológicos e históricos) para la cuenca superior y sobre la actividad agrícola tradicional para el eje de crecimiento oeste de la aglomeración del Gran Buenos Aires.

Los objetivos generales a nivel cuenca son: (1) realizar un diagnóstico poblacional de sus municipios, y (2) determinar la distribución espacial de las áreas de potenciales conflictos entre usos del suelo urbano, agrícola y de conservación ambiental. 
Otros objetivos de importancia se focalizan en sectores de la cuenca: (1) determinar y analizar la expansión urbana en los partidos de la cuenca superior y evaluar el riesgo que representa para los sitios arqueológicos (históricos y prehispánicos) de interés patrimonial y (2) determinar y analizar la expansión urbana en los partidos de la del sector de crecimiento oeste de la aglomeración Gran Buenos Aires a fin de evaluar su impacto sobre la productividad tradicional.

La investigación tuvo claramente una focalización espacial a partir de apoyarse en los cinco conceptos centrales del análisis espacial analizando aspectos de localización, distribución espacial, asociación espacial, interacción espacial y evolución espacial; para lograrlo se basa en una perspectiva científica de carácter sistémico apoyada en el uso de métodos cuantitativos y cartografía digital trabajada mediante vinculaciones geoinformáticas (Buzai et al., 2016), una perspectiva de gran dinamismo actual.

\section{Perspectiva científica}

La Geografía considerada como ciencia espacial tiene como marco general la filosofía positivista, la cual tiene entre sus postulados el criterio demarcatorio producido por el monismo metodológico, esto es, que toda la ciencia debe utilizar el denominado método científico como una forma específica de aproximación para la construcción de conocimientos.

Es una metodología basada en una aproximación racional hacia la realidad empírica y estandarizada para todo campo de conocimiento. Según Russell (1985) su procedimiento se basa en la observación de los hechos significativos, la generalización de las observaciones para la formulación de leyes y modelos, y finalmente la comprobación que puede ser realizada en nuevos casos que propone la realidad.

En base a estas consideraciones, generalmente se afirma que la experimentación como método central de muchas ciencias naturales es imposible llevarla a cabo en las ciencias sociales. Sería imposible poner aspectos sociales en un laboratorio o en un tubo de ensayo... Sin embargo, una de las ciencias naturales de mayor prestigio es la Astronomía, una ciencia que construyó sus bases a partir de mediciones obtenidas a partir de la observación sistemática y no del experimento (Buzai, 2016). De esta manera se considera que las ciencias fácticas son empíricas, porque parten de los hechos de la realidad a través de la observación de temas y problemas, y cuando se hace ciencia aplicada se vuelve a la realidad para actuar sobre ella.

La Geografía como ciencia espacial, basada en la cuantificación, observa la realidad empírica del espacio geográfico, realiza mediciones de esa realidad, analiza las distribuciones espaciales y las generaliza a través de modelos y la formulación de leyes.

Mediante este accionar se amplía su definición, y a las tradicionales de tipo ecológico (estudio de la relación hombre-medio/sociedad-naturaleza) y corológico (estudio de la diferenciación areal), se le suma una definición sistémica como la "ciencia que formula y aplica las leyes que rigen las pautas de distribución en el espacio geográfico".

En este contexto, en donde queda privilegiada la observación, la medición y la búsqueda de regularidades de las distribuciones espaciales, el mapa se convierte en una herramienta funda- 
mental que permite dar soporte a una gran cantidad de procedimientos metodológicos de la disciplina.

\section{Aproximación sistémica}

La estructura y dinámica de las distribuciones espaciales no se producen de manera aleatoria, sino que pueden entenderse a través de poner en evidencia las leyes que rigen las pautas de distribución espacial. Estas leyes surgen a partir de estudiar comportamientos generales empíricos y son utilizadas para realizar predicciones teóricas de las configuraciones futuras en un claro accionar predictivo de la investigación científica.

Investigaciones realizadas en diferentes campos del conocimiento durante el siglo XX fueron transformando las perspectivas iniciales de cada especialización al centrarse en el estudio de los objetos materiales y relaciones en componentes que muchas veces se consideraron aislados. La ciencia comenzó a ver totalidades a través de considerar la realidad como sistema.

La Teoría General de los Sistemas (TGS), fue enunciada por Von Bertalanffy (1988) como teoría global que sustenta una perspectiva modelística en elementos y relaciones. Su objetivo central es determinar similitudes estructurales entre fenómenos comunes de diferentes realidades empíricas $y$, de esta manera, poder utilizar las mismas herramientas para analizar estructuras diferenciadas según el campo de aplicación. Una misma función matemática podría utilizarse para describir comportamientos de células, poblaciones humanas, ciudades o cuerpos celestes.

Como parte de una estructura cada elemento encuentra su explicación en relación a otros elementos y con la estructura total que los vincula. Se considera que la perspectiva sistémica permitió unir las piezas disgregadas surgidas de la alta especialización y brinda perspectivas globales al enfoque analítico.

La aplicación de la TGS en el análisis espacial permitirá obtener resoluciones a través del uso de las matemáticas como lenguaje de la ciencia y de la geometría como el lenguaje de las formas espaciales (Haggett, 1977). La perspectiva sistémica permitirá unir las escalas (Bunge, 2003) entre el detalle abordado por las aproximaciones atomistas y las generalizaciones del holismo.

Si se pone atención sobre aspectos específicos es posible focalizarse en diferentes escalas en la búsqueda de una complementación que fue lograda con el desarrollo de la Teoría de los Sistemas Complejos (TSC) sistematizada por García (2006) como sustento del trabajo científico interdisciplinario a partir de las bases propuestas por la Epistemología Genética.

La TSC ha demostrado grandes capacidades para el estudio de la realidad socioespacial en las investigaciones geográficas y, también, para entender aspectos correspondientes a la construcción de conocimientos en base a su aptitud epistemológica.

Considera que la realidad se encuentra formada por una estructura estratificada a partir de escalas con organizaciones semi-autónomas. Al aplicar la TSC al análisis espacial se realizará un estudio en un nivel de análisis específico (nivel focal), uno superior (nivel suprafocal) y otro inferior (nivel infrafocal) poniendo atención en las dinámicas específicas de cada escala. Esta situación 
permite ampliar la TGS contando con un marco general que permite sustentar la estabilidad de determinadas teorías en sus niveles específicos.

La TGS se focaliza en aspectos generales y la TSC considera especificidades. Ambas brindan la posibilidad de estudiar la realidad como totalidad en sus múltiples dimensiones y detalles. En este esquema, toda representación cartográfica se encuentra ubicada en el nivel focal espacial como meso-escala y a partir de allí la diferenciación espacial de sus categorías encontrará explicación en diferentes niveles de análisis sintetizados en la Geografía como ciencia espacial.

\section{Contexto tecnológico}

El contexto tecnológico de la investigación se enmarca en el concepto de Geoinformática representado en la Figura 3. El trabajo cartográfico tuvo una extensa etapa analógica en la cual todo el trabajo era realizado en papel (era geoanalógica) y a partir de la década de 1960 comenzó a transitarse el camino de la digitalización a partir del cual comenzó a considerarse la existencia de una cartografía automatizada (era geodigital).

La primera etapa duró poco más de 2.700 años al considerar el primer mapa del mundo realizado por Anaximandro en el siglo VIII a.C. (Buzai, 2016) y la etapa actual apenas supera los 50 años (Buzai, 2015a) y según Rabella (2016) se presenta como una nueva dimensión para el trabajo cartográfico explotando todas sus posibilidades en las tareas del análisis espacial.

El primer software SIG (Canada Geographical Information Systems - GSIG, 1964) hace que esta tecnología tenga actualmente 54 años de existencia. Su evolución en muchas líneas de análisis (Buzai y Robinson, 2010) permitió un amplio reconocimiento de la componente espacial por parte de variadas disciplinas y múltiples aplicaciones.

Toda entidad geográfica comenzó a ser modelada mediante dos componentes: forma y contenido. Desde un punto de vista informático a partir de la creación de bases de datos gráficas y bases de datos alfanuméricas.

Las formas son representaciones geométricas (punto, línea, polígono, celda raster o $x$-tree) que pueden ser trabajadas mediante el siguiente software: Diseño Asistido por Computadora (CAD, Computer Aided Design), Cartografía asistida por computadora (CAC), Gestión de infraestructura (AM-FM, Automated Mapping-Facilities Management), Sistemas catastrales (LIS, Land Information Systems), Procesamiento digital de imágenes satelitales (PDI) y Modelos digitales de elevación - 3D (DEM, Digital Elevation Models).

Los contenidos son principalmente mediciones cuantitativas que se incorporan en la tabla de atributos asociada a la gráfica que pueden ser tratados mediante el siguiente software: Editores de textos (EDT), Administradores de bases de datos (ABD), Planillas de cálculo (PLC), Programas de análisis estadístico (PAE) y Sistemas de posicionamiento global (GPS).

La convergencia de software muestra al SIG como núcleo de la Geoinformática. Esta convergencia permitió la aparición de la "Geografía Automatizada" (Dobson, 1983) ampliamente desarrollada a través de los Sistemas de Ayuda a la Decisión Espacial (SADE). Se generan las bases 
Figura $\mathrm{N}^{\circ} 3$

Geoinformática como contexto técnológico de la investigación

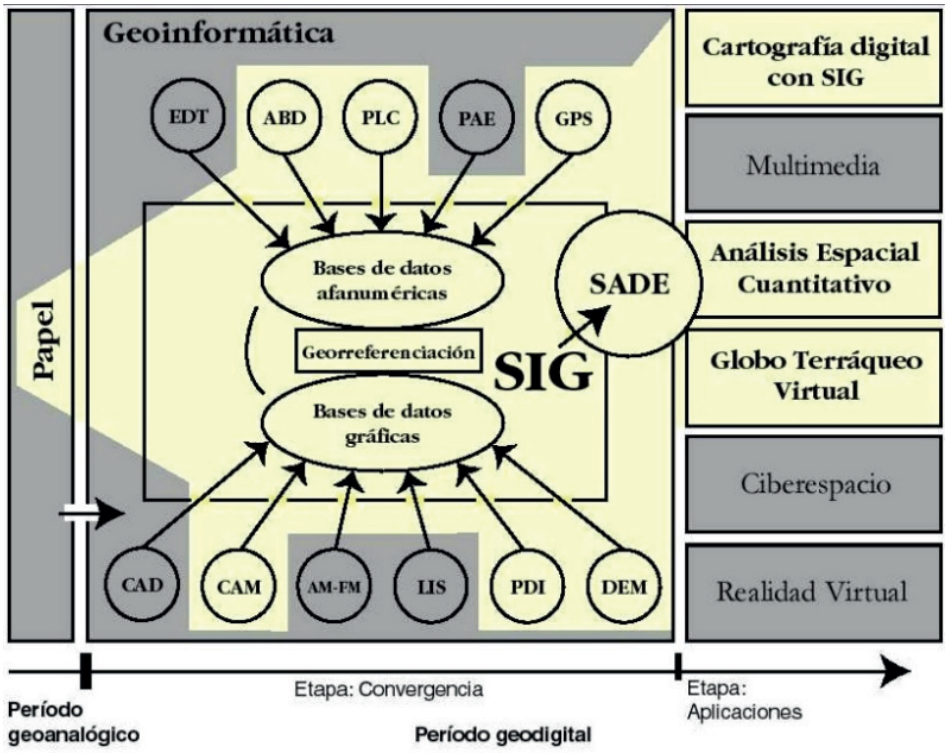

Fuente: Elaboración propia a partir de Buzai y Baxendale (2011)

para una expansión de impacto científico a través de la Geografía Global y una de impacto social a través de la Neogeografía (Buzai, 2014).

En la Figura $\mathrm{N}^{\circ} 3$ queda representado en tonalidad clara el contexto tecnológico que hemos utilizado en la presente investigación hacia el logro de los objetivos técnicos planteados. Las tareas realizadas en el proyecto incluyeron trabajos con mapas en papel, creación de base de datos gráfica mediante programas CAC, utilización de datos censales mediante ABD y PLC, delimitación de la cuenca mediante PDI, trabajo de campo apoyado con GPS y resultados en la línea de la Cartografía digital con SIG, vinculación de la cartografía al globo terráqueo virtual (GTV) como los proporcionados por Google Earth o Bing Maps y procedimientos del análisis espacial cuantitativo, algunos de ellos apoyados por Sistemas de Ayuda a la Decisión Espacial (SADE).

\section{Delimitación regional}

\section{Delimitación inicial (2002)}

Hace más de una década el Laboratorio de Cartografía Digital (LaCaD) del Departamento de Ciencias Sociales de la Universidad Nacional de Luján publicaba dos atlas digitales (Buzai, 2001, 2002) con finalidad didáctica orientada a la enseñanza secundaria. El segundo lleva como título "Atlas digital de la cuenca del río Luján - Socio-demográfico, económico y ambiental" y en versión CD incluyó los archivos shape (.shp) de SIG junto con el visualizador ArcView 1.0 y un sintético manual del usuario. 
El espacio geográfico del área de estudio se encontraba completamente contenido en la carta (topográfica e imagen) "San Martín" de escala 1:250.000 editada por el Instituto Geográfico Nacional (IGN) con la nomenclatura 3560-II (Figura № 4-A). A partir de ella se realizó la digitalización mediante tableta digitalizadora y el espacio regional fue delimitado a partir del cálculo de los puntos medios de los diferentes grandes sistemas fluviales dentro de los partidos que los contienen. Como resultado de la delimitación fue definida una cuenca de $2.900 \mathrm{~km}^{2}$ de superficie (Figura $\mathrm{N}^{\circ} 4-\mathrm{B}$ ).

La cuenca del río Luján se encuentra contenida en 14 unidades espaciales correspondientes a los municipios de Campana, Escobar, Exaltación de la Cruz, General Rodríguez, José C. Paz, Luján, Malvinas Argentinas, Mercedes Moreno, Pilar, San Andrés de Giles, San Fernando, Suipacha y Tigre.

Figura $\mathrm{N}^{\circ} 4$

Cuenca del río Luján, 2002

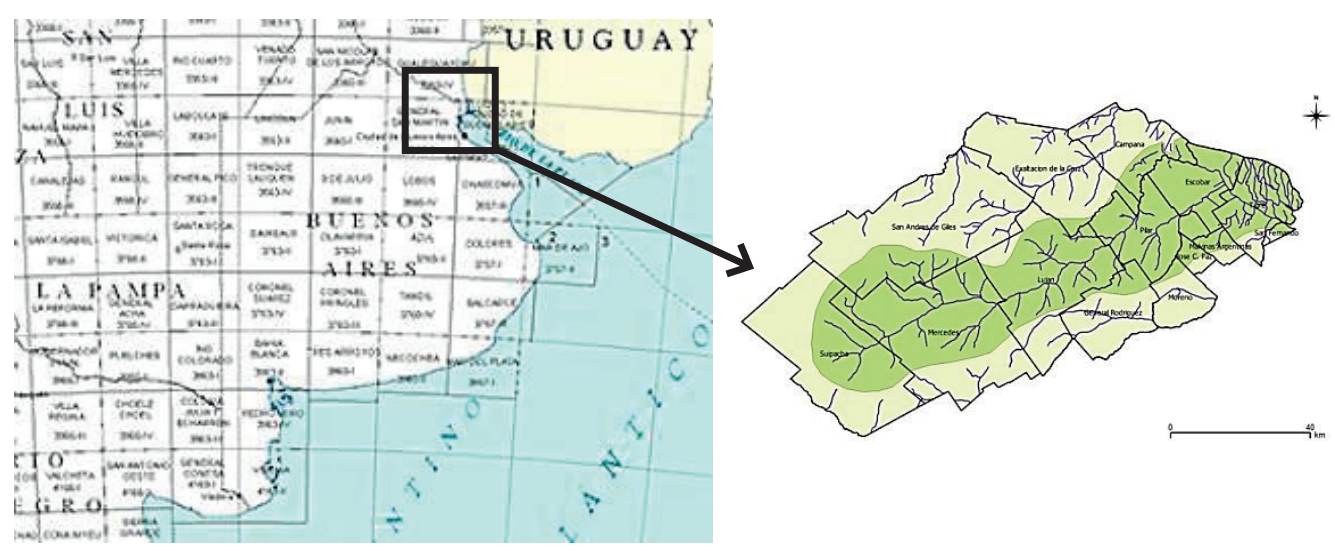

A. Ubicación de cartas topográficas IGN en esca-

B. Cuenca del río Luján (2002). Situación relativa las 1:250.000 en la Provincia de Buenos Aires. correspondiente a la digitalización cartográfica.

\section{Delimitación actual (2015)}

1. Uso de cartografía topográfica con mayor detalle: ajustes en la cuenca superior y actualización de los límites

La realización del proyecto tuvo como primera tarea técnica realizar la definición espacial del área de estudio a partir de ajustar el área obtenida en el año 2002 mediante la utilización de mapas topográficos con mejor resolución espacial. En este caso la finalidad es contar con una base cartográfica destinada a la investigación científica con extensión hacia las prácticas de ordenamiento territorial.

Los límites regionales representados en la Figura $\mathrm{N}^{\circ}$ 4-B obtenidos de la generalización realizada en escala 1:250.000 fueron analizados con mayor detalle a partir de las cartas topográficas 
oficiales en escala 1:50.000 con las siguientes cartas: Castilla, Estación Román Báez, Franklin, Heavy, Luján, Mercedes, Moreno, Open Door, Pilar, Plomer, San Andrés de Giles, San Fernando, Suipacha y Tomás Jofré.

A partir de estas cartas fueron reconocidas amplias zonas de humedales que permitieron ampliar el área de estudio, principalmente en la cuenca superior incorporando 2 municipios a los 14 considerados inicialmente. Se incluyen Carmen de Areco y Chacabuco.

La Figura $N^{\circ} 5$ presenta parte del trabajo manual realizado sobre el cuadrante inferior izquierdo de la carta topográfica Suipacha (3560-16-2), en la cual el trazado de una doble línea con color interior muestra el nuevo límite obtenido a partir de los valores de las cotas máximas, la Figura 6 presenta el resultado del trabajo de digitalización mediante CAC para la cuenca superior, y finalmente en la Figura $\mathrm{N}^{\circ} 9$ fueron llevados los resultados a la escala 1:250.000 donde queda definido el ajuste que actualiza el área de estudio total.

Figura $\mathrm{N}^{\circ} 5$

Detalle (cuadrante inferior izquierdo) de la carta topográfica Suipacha

(3560-16-2), escala 1:50.000

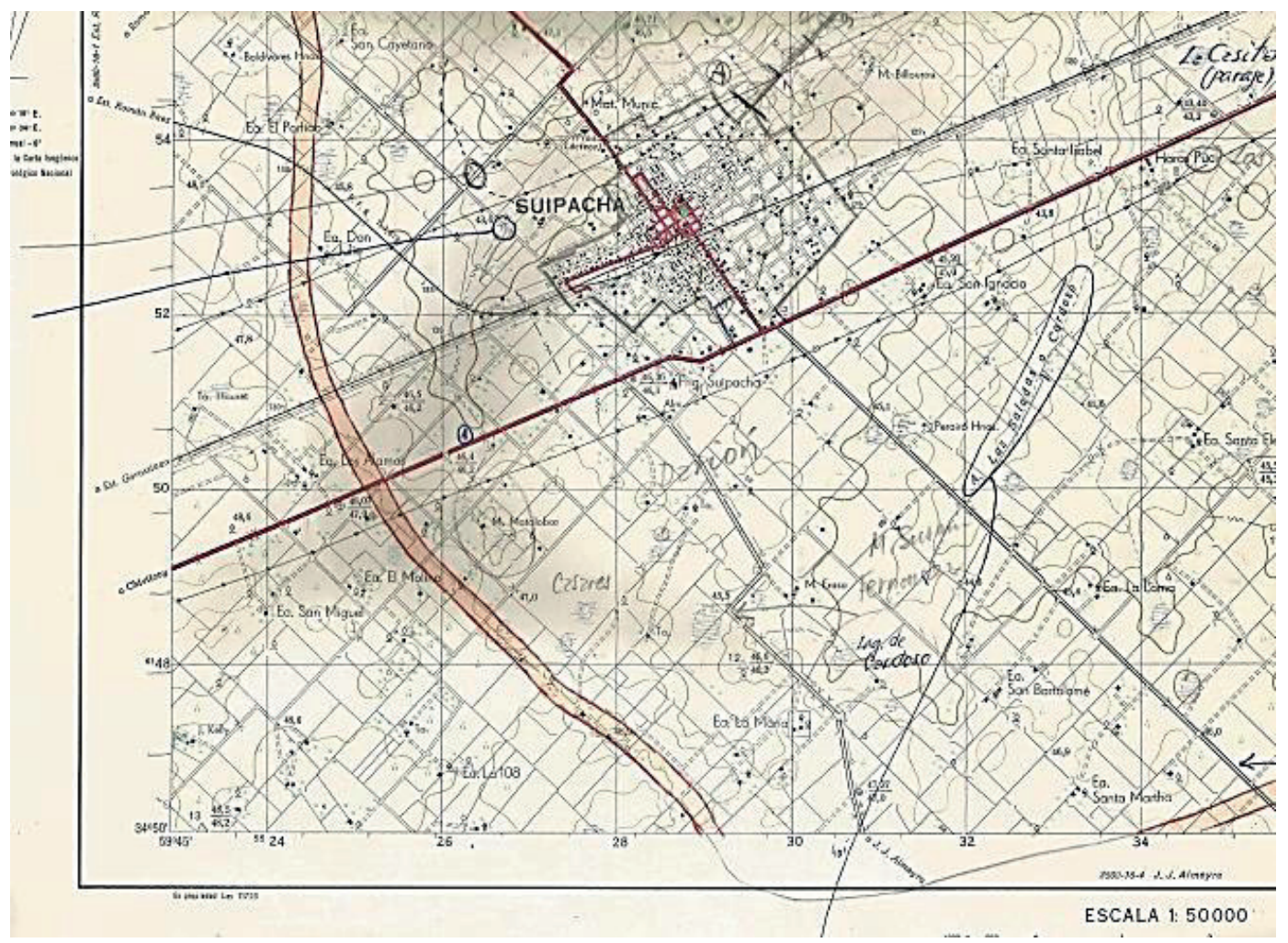

Fuente: Elaboración propia. 
Figura $N^{\circ} 6$

Límite de la cuenca superior en el Partido de Suipacha

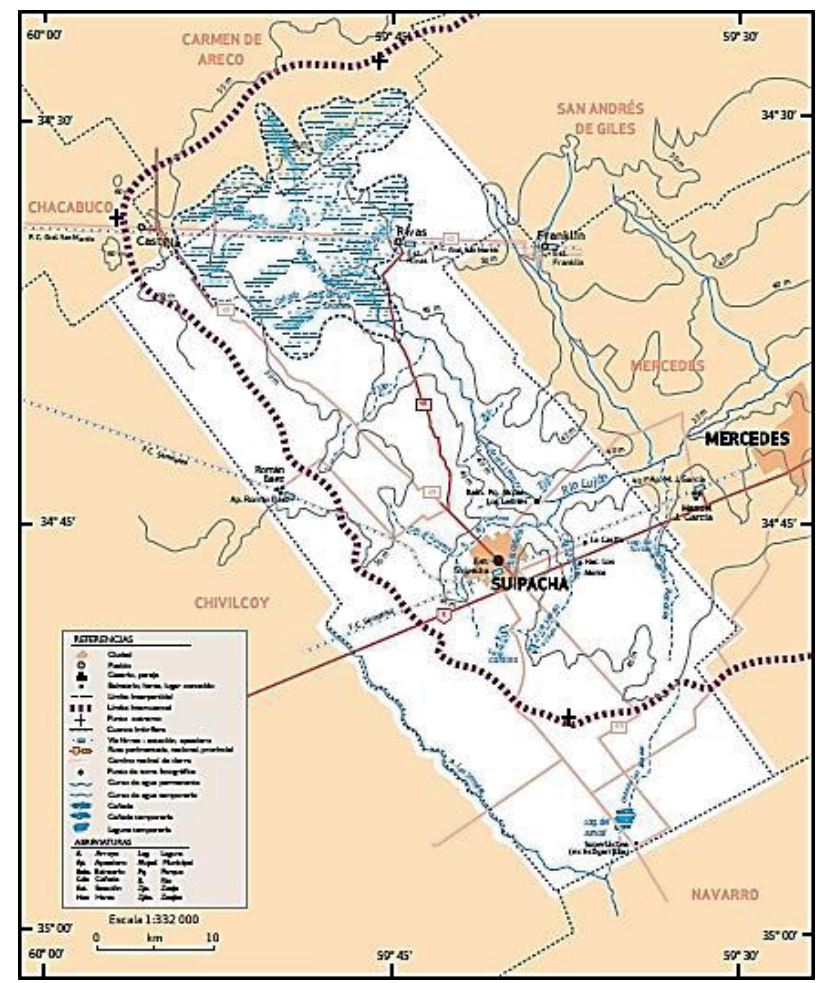

Fuente: Elaboración propia.

Figura $\mathrm{N}^{\circ} 7$

Arroyo del Durazno

(naciente del río Luján en Suipacha)

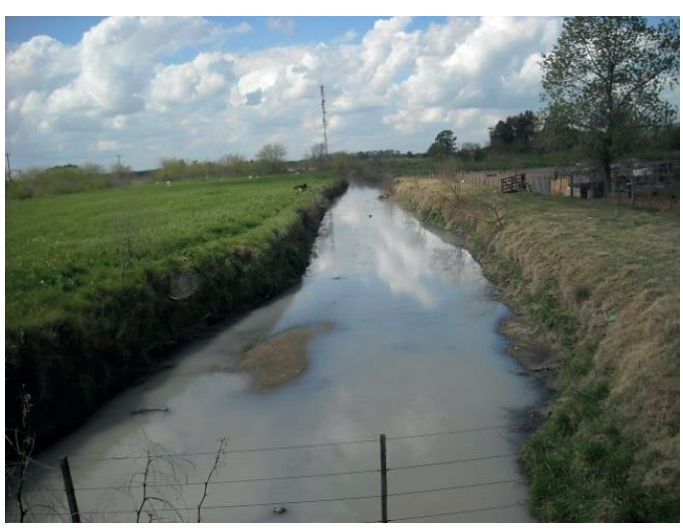

Fuente: Archivo fotográfico personal de Luis Fernando Paso Viola.
Figura $\mathrm{N}^{\circ} 8$

Arroyo Cañada de Rivas

(naciente del río Luján en Suipacha)

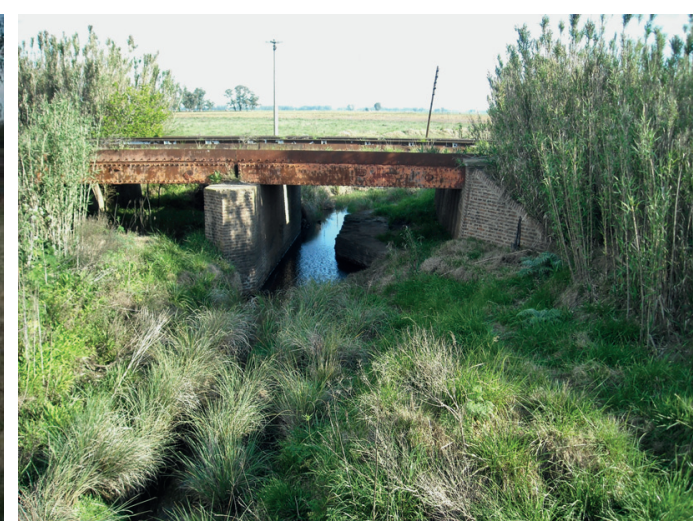

Fuente: Archivo fotográfico personal de Luis Fernando Paso Viola. 
Figura $\mathrm{N}^{\circ} 9$

Carta topográfica San Martín (3560-II), escala 1:250.000

(Límites ajustados de la cuenca del río Luján)

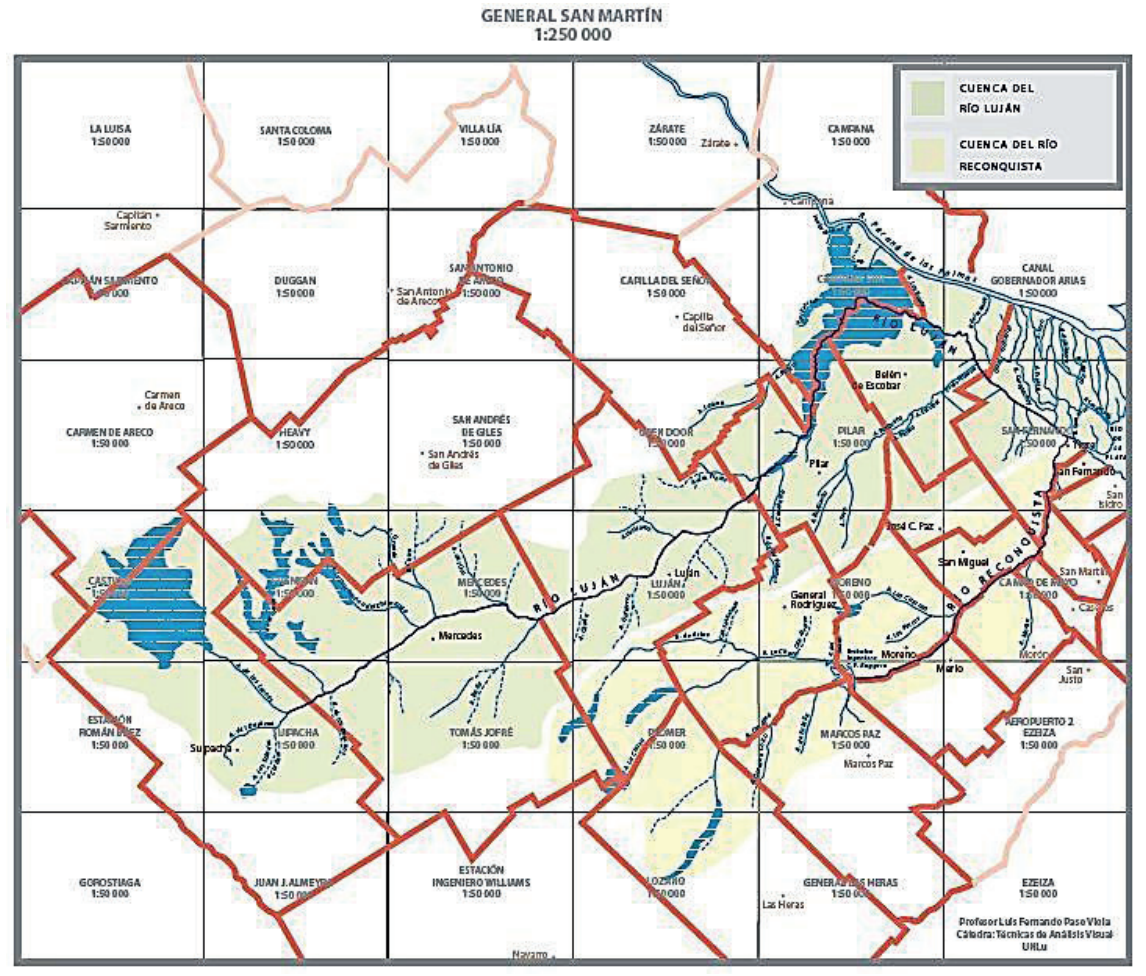

Fuente: Elaboración propia.

\section{Procesamiento digital de imágenes}

El mapa presentado en la Figura $\mathrm{N}^{\circ} 9$ corresponde a la delimitación cartográfica realizada mediante el análisis visual de mapas topográficos para la delimitación completa del área de estudio. Las modificaciones generan ajustes a la cartografía realizada en el Proyecto Atlas. En esta instancia se presta particular atención a los límites de la cuenca superior en base a los trabajos realizados por Paso Viola (2013) y al extremo Este (Lanzelotti y Buzai, 2015), su desembocadura compartiendo con el delta del río Paraná diversas condiciones físico-naturales.

La actual definición de mayor claridad en el ajuste de sus límites expande el área de estudio a una superficie de $3.778 \mathrm{~km}^{2}$.

El río Luján nace de la confluencia de las cañadas de Castilla, Rivas y otras como arroyo de Los Leones por lo que su cuenca de alimentación atraviesa territorios de los partidos de Chacabuco y Carmen de Areco, siendo que la extensión de esta última hace incorporar una pequeña superficie del Partido de Chacabuco. Su recorrido tiene una dirección general SO-NE y una longitud de $115 \mathrm{~km}$, hasta desembocar en el delta del Parará. A partir de allí continúa su curso en dirección 
noroeste-sudeste hasta llegar al río de La Plata, formando, en este último tramo de $42 \mathrm{~km}$, parte de la dinámica del delta.

Un avance en la metodología que incorpora la automatización digital se basa en el procesamiento computacional de modelos digitales de elevación (DEM) posibilitado a partir de imágenes satelitales.

Para realizarlo se utilizaron imágenes satelitales SRTM y ASTERGDEM ${ }^{6}$ como insumo procesadas con SIG. ${ }^{7}$ A partir de estos resultados la definición espacial se corrigió mediante interpretación visual de imágenes realizadas con las cartas topográficas tanto en el límite oeste de la cuenca superior, como en su sección del delta del Paraná en la cuenca inferior.

Se comprobó que las dos imagenes satelitales que mejor representaron la superficie de esta parte del país corresponde a la N SRTM_ffB03_p225r084.tif proyectada en UTM faja 21 y la imagen satelital GLSDEM_p225r84z2111 (Figura $N^{\circ} 10$ y Figura $N^{\circ} 11$ ).

Figura $\mathrm{N}^{\circ} 10$

Superficie de la cuenca del río Luján sobre la imagen satelital RTM_ffB03_p225r084

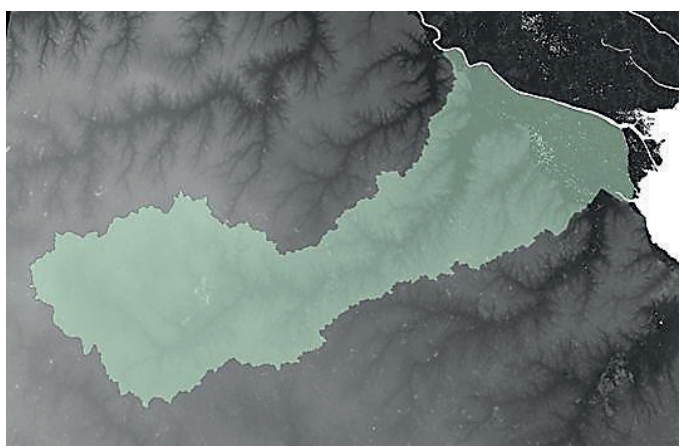

Fuente: Elaboración propia.
Figura $N^{\circ} 11$

Superficie de la cuenca del río Luján sobre la imagen satelital GLSDEM_p225r84z2111

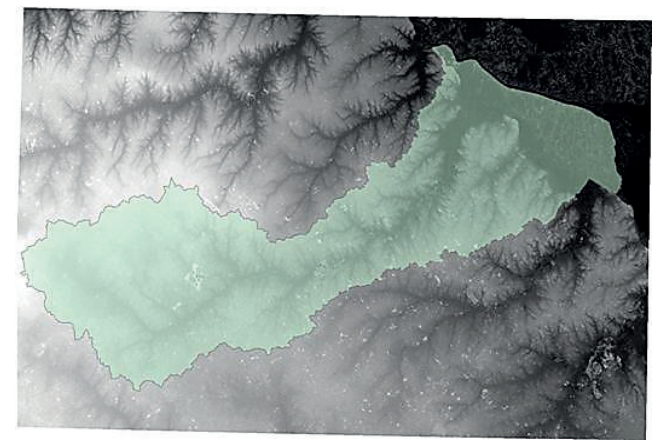

Fuente: Elaboración propia.

La Figura № 12 presenta el resultado de la definición espacial con la capa temática de municipios superpuesta. Comparada con la Figura $4 \mathrm{~b}$ se aprecia su mejor definición en sus límites y con la Figura 6 una gran correspondencia en la ampliación de su superficie y la consideración de dos partidos más en su nacimiento. 
Figura $\mathrm{N}^{\circ} 12$

Superficie de la cuenca del río Luján sobre la imagen satelital BING

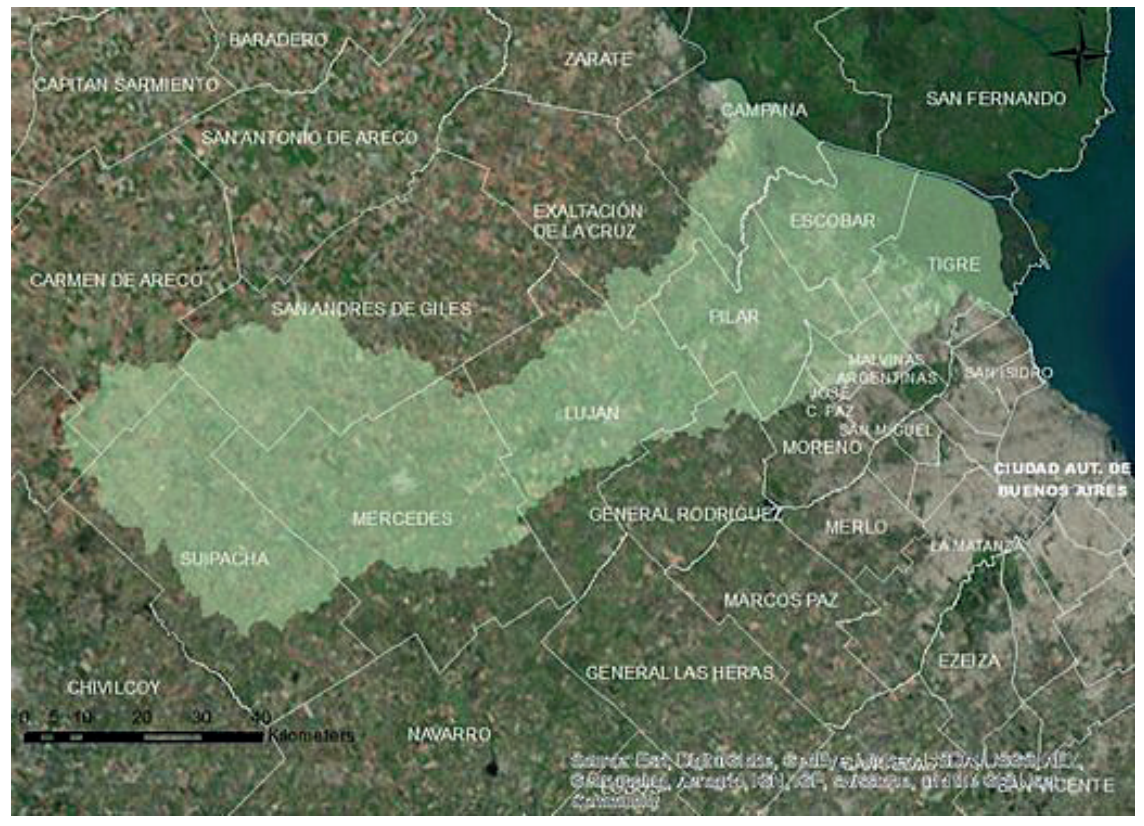

Fuente: Elaboración propia.

De esta manera adoptamos la definición espacial obtenida a través del DEM ya que pudo ser claramente validada utilizando los ajustes obtenidos en el trabajo cartográfico de detalle.

Una vez determinados los límites de la cuenca pueden definirse sus principales áreas internas y las divisiones político-administrativas que la contiene y considerar las unidades espaciales de captación de datos censales. De esta manera la base cartográfica en SIG queda conformada por dos capas temáticas, una utilizada para las aplicaciones en sistema raster y otra para las vectoriales y censales.

De acuerdo al INA (2007) la cuenca puede dividirse en tres tramos: (1) Cuenca Superior: se extiende desde las nacientes hasta la localidad de Jáuregui localizada a $7 \mathrm{~km}$. al Oeste de la ciudad de Luján. Presenta una pendiente media del tramo de unos 0,45 m/km., (2) Cuenca Media: desde la localidad de Jáuregui hasta las proximidades del cruce con la Ruta Nacional $N^{\circ} 8$, en la localidad de Pilar. Con una pendiente media del tramo de 0,36 m/km. y (3) Cuenca Inferior: desde las inmediaciones del cruce con la Ruta Nacional No 8 hasta su ingreso del Delta del Paraná, poco después del cruce de la Ruta Nacional No 9. La pendiente media en este tramo es del orden de 0,04 m/km.

De acuerdo al INDEC (2010) la República Argentina se divide en unidades espaciales de diferente orden: (1) País, (2) Provincias, (3) Partidos -nivel municipal en la Provincia de Buenos Aires-, (4) Fracciones censales y (5) Radios censales. Mientras que las 1, 2 y 3 corresponden a diferentes instancias de gobierno, las 4 y 5 son unidades de captación de datos con mayor detalle. 
La Figura No 13 presenta la base cartográfica en SIG compuesta por dos capas temáticas: (1) cuenca del río Luján con sus sectores, y (2) división político-administrativa de municipios y unidades censales de fracciones censales.

Figura $N^{\circ} 13$

Base cartográfica

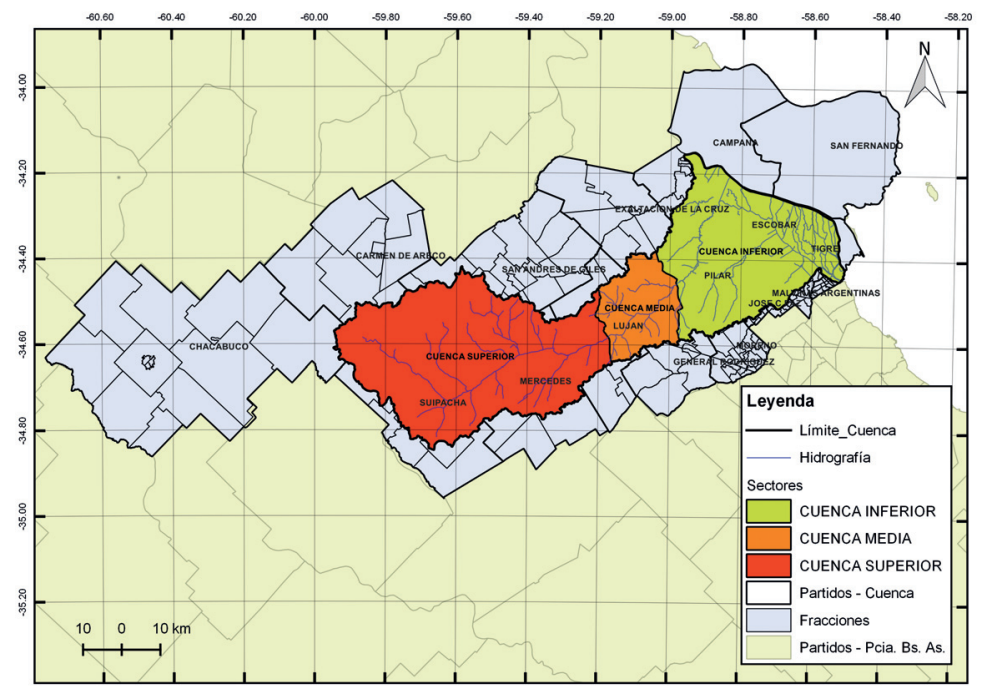

Fuente: Elaboración propia.

\section{Geografía cuantitativa y cartografía temática}

A partir de la definición del área de estudio fueron confeccionadas las bases cartográficas digitales para el análisis de diferentes temáticas en su interior, principalmente el estudio de la distribución espacial de la población y de los usos del suelo urbano, agrícola y de conservación. Ambas líneas corresponden a los objetivos generales a nivel cuenca y en este punto serán presentadas dos síntesis geográficas de las distribuciones espaciales resultantes.

\section{Cartografía temática vectorial: clasificación espacial}

Los procedimientos en SIG vectorial se orientan a la realización de cartografía temática univariada, el análisis exploratorio de datos espaciales (ESDA, Exploratory Spatial Data Analysis) y síntesis socioespaciales a partir de la aplicación de métodos de análisis multivariado.

En este caso fueron combinadas 5 dimensiones a través de una variable representativa para cada una de ellas (se presentan los valores mínimos y máximos que tienen las fracciones censales): (1) Educativa (Nivel educativo: Universitario completo, 0-3375), (2) Pobreza (Hogares con Necesidades Básicas Insatisfechas, 0-4148), (3) Vivienda (Viviendas con piso de cerámica, 8-5243), (4) Servicios (Hogares con agua por red, 8-6246) y (5) Equipamiento (Hogares con computadoras, 7-13875). 
Con la finalidad técnica de lograr la comparabilidad perfecta entre las variables fueron estandarizadas en puntajes omega $(\Omega)$ multiplicado por 100, en este sentido los valores originales se trasladan a un rango 0-100.

Las variables 1, 3, 4 y 5 son consideradas de beneficio, ya que en sus máximos puntajes indican situaciones favorables. En ellas se aplica el cálculo directo:

$$
\text { [ 4 ], } \Omega=\frac{x-m}{M-m} \times 100 \text {, }
$$

donde $x$ es el valor de la variable en cada unidad espacial, $M$ y $m$ corresponden a los valores mayor y menor de la serie de datos.

La variable 2 es considerada de costo, ya que en sus máximos puntajes indica situaciones desfavorables. En ellas se aplica el cálculo inverso a fin de orientarla en el sentido de las variables de beneficio:

$$
\text { [ 5 ], } \Omega=\frac{M-x}{M-m} \times 100 \text {, }
$$

Finalmente, el puntaje de clasificación espacial (PCE) está formado por el promedio de las cinco variables estandarizadas de acuerdo al esquema metodológico de la Figura 14 y se interpreta en el sentido de los mapas de beneficio.

$$
\text { [6], } P C E=\frac{\sum \Omega}{N}
$$

Figura $\mathrm{N}^{\circ} 14$.

Esquema metodológico de resolución cuantitativa hacia la obtención del puntaje de clasificación espacial (PCE)

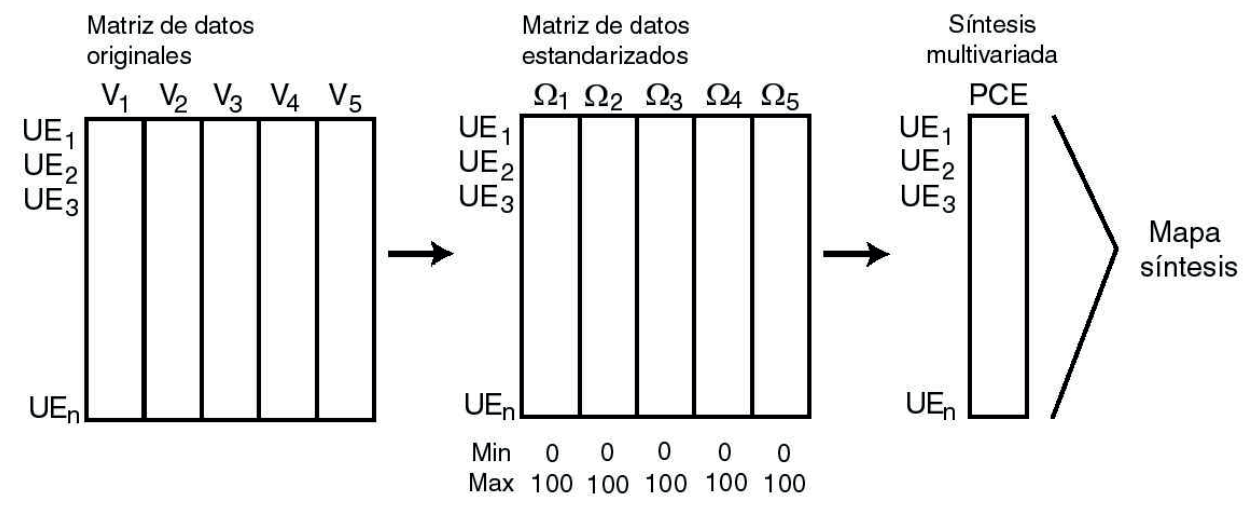

Fuente: Elaboración propia. 
La Figura $N^{\circ} 15$ presenta los resultados correspondientes a la distribución espacial del PCE compuesto por la combinación de las variables estandarizadas en cortes naturales con cinco intervalos de clase.

\section{Figura $N^{\circ} 15$}

Cuenca del río Luján. Mapa social por clasificación espacial

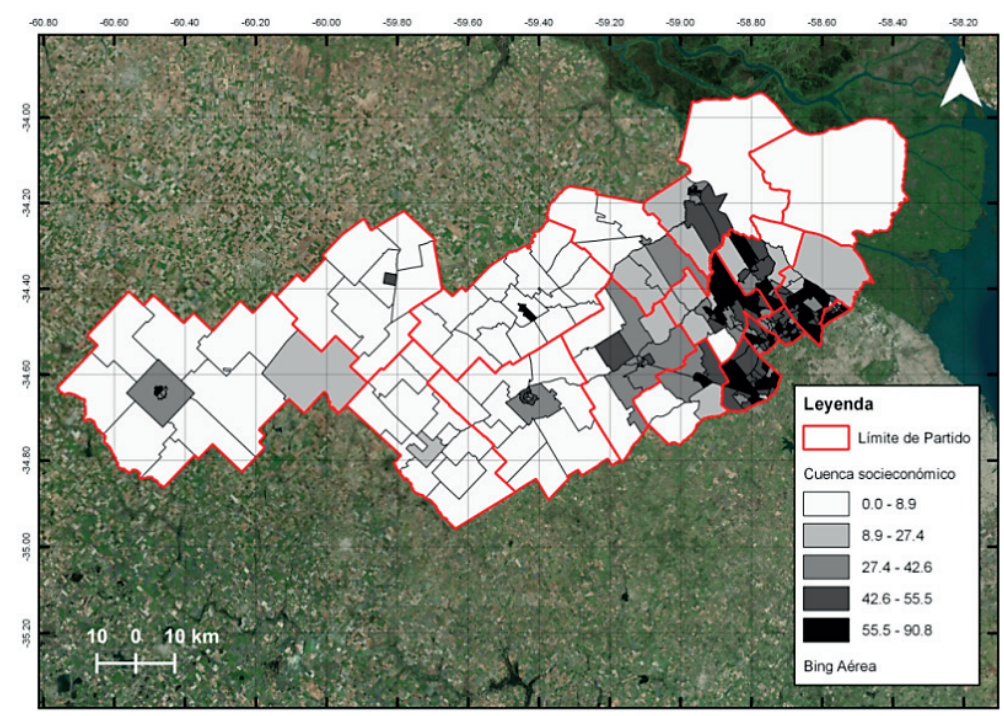

Fuente: Elaboración propia.

El resultado define un mapa social del espacio regional a partir de variables sociodemográficas representadas en base a las fracciones censales de los municipios del área de estudio (Buzai y Baxendale, 2017). En este mapa queda evidente que los mayores valores están vinculados a las áreas urbanas de expansión del Gran Buenos Aires en la cuenca inferior y en los principales núcleos urbanos de ciudades de tamaño intermedio en la cuenca media y superior.

\section{Cartografía temática raster: modelado cartográfico}

Los procedimientos en SIG raster se orientan a la representación de variables correspondientes a la realidad socioambiental y al análisis de usos del suelo mediante el modelado cartográfico y las técnicas de evaluación multicriterio.

La Figura 16 presenta el esquema metodológico utilizado para la resolución mediante las técnicas del modelado cartográfico, evaluación multictriterio y LUCIS (Land Use Conflict Idenfiction Strategy) (Buzai y Baxendale, 2011).

El resultado tiene su base en una combinación lineal ponderada (CLP)

$$
\text { [1] } C L P=\sum x p \prod r
$$

Donde $x$ es cada capa temática, $p$ los valores de ponderación y $r$ los mapas de restricciones booleanas. Las ponderaciones cumplen la siguiente propiedad: 


\section{[2] $0<p \leq 1$ \\ [3] $\sum p=1$}

Figura $\mathrm{N}^{\circ} 16$

Esquema metodológico de resolución cuantitativa en combinación de la metodología de evaluación multicriterio y LUCIS

EVALUACIÓN MULTICRITERIO

Fuente: Buzai y Principi (2017).

Figura $\mathrm{N}^{\circ} 17$

Cuenca del río Luján. Potenciales conflictos entre usos del suelo

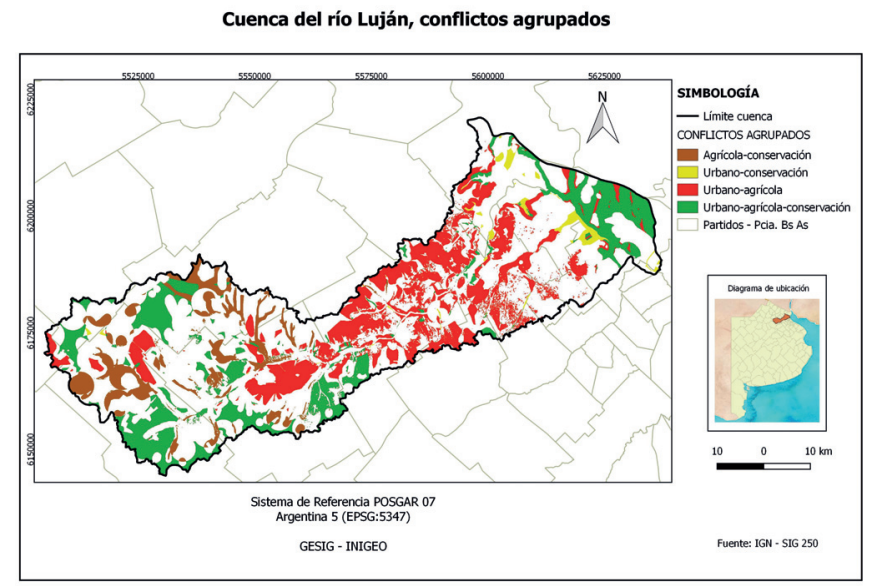

Fuente: Elaboración propia. 
La Figura $N^{\circ} 14$ corresponde a los resultados prospectivos de potencialidad de conflictos ante la evolución espacial de usos del suelo correspondientes a mapas de aptitud para las coberturas urbanas, de agricultura y para conservación (Buzai y Principi, 2017).

Los resultados obtenidos en el cálculo de superficie de las categorías de la cartografía temática indican que las áreas de potenciales conflictos entre usos del suelo en la cuenca del río Luján estarían definidas de la siguiente manera: Agrícola-Conservación $(252,26$ km²), Urbano-Conservación (49,62 km²), Urbano-Agrícola $(712,03$ km²) y Urbano-Agrícola-Conservación (564,03km²), en total la potencialidad de conflictos completos es de $1577,94 \mathrm{Km}^{2}$ lo que corresponde a un $49,95 \%$ de la superficie de la cuenca.

\section{Consideraciones finales}

A lo largo del trabajo hemos presentado los desarrollos cartográficos realizados en un proyecto de investigación científica y tecnológica centrado en el estudio de problemáticas de naturaleza espacial. La representación espacial en diferentes instancias y ante la utilización de diferentes recursos (cartas topográficas, cartografía digital e imágenes satelitales) marcan un hilo conductor hacia la obtención de los resultados presentados a través de la cartografía temática.

El uso cartográfico mostró utilidad en la totalidad de instancias de la investigación. Al inicio para definir el área de estudio, durante su desarrollo en la sistematización de datos y finalmente, vinculado a la Geografía Cuantitativa mediante el uso de SIG, hacia la obtención de los resultados parciales y finales de la investigación.

Al centrarnos en la metodología claramente queda demostrado que en la relación entre Geografía y Cartografía se hacen operativos aspectos teóricos apoyados en una serie de conceptos centrales de naturaleza espacial.

Surgidos de los casos de aplicación presentados en este trabajo, serán considerados los conceptos centrales de localización, inventario y análisis prospectivo que González Leiva (1984) consideró como centrales ante la evolución cartográfica a partir de la automatización digital, y los complementamos con conceptos del análisis espacial como los de distribución espacial, asociación espacial y síntesis (Buzai, 2015b).

La localización se hace operativa ante la ubicación que cada unidad espacial ocupa sobre la superficie terrestre, corresponde al simple hecho de considerar cada entidad geográfica en el interior de un sistema de coordenadas, aquí se encuentran las celdas raster o las entidades vectoriales asociadas a sus atributos. Todo dato asociado espacialmente puede consultarse en un SIG a través de la herramienta de interrogación.

La "distribución espacial" presenta en el sistema raster o la conformación de intervalos de clase en un conjunto de unidades espaciales poligonales en sistema vectorial. En este segundo caso el SIG toma los datos de la base de datos alfanumérica asociada y los distribuye en el área de estudio utilizando determinadas variables visuales a partir del método clasificatorio adoptado. Este es uno de los conceptos centrales de la Geografía, ya que apoya su definición corológica y el lenguaje cartográfico se torna central al mostrar diferencias culi-cuantitativas en distintos espacios. 
El "inventario" experimentó una importante modificación ante el uso geoinformático ya que ahora no se lo debe buscar en el mapa, sino que pasa a estar en la geometría de diversas capas temáticas y en estructuras matriciales separadas de las bases cartográficas y esto, en teoría, lo hace infinito. En este esquema relacional el inventario brinda los datos necesarios para cada trabajo específico que lleve a la realización de mapas de tratamiento y síntesis.

El "tiempo", cuando la investigación avanza hacia el futuro, hace que la cartografía pueda ser de utilidad para trabajos prospectivos como la búsqueda de sitios con potencialidad para encontrar localizaciones óptimas o zonas de conflictos espaciales. Permiten estimarse escenarios de cambio en una proyección futura espacio-temporal. En este caso el modelado cartográfico y las técnicas de evaluación multicriterio presentan el camino de resolución, pero otras importantes posibilidades han sido automatizadas mediante el uso del SIG, como las ventanas móviles y uso de autómatas celulares (Buzai y Baxendale, 2011).

La "asociación" espacial está relacionada a la superposición cartográfica que contiene diferentes distribuciones espaciales. El procedimiento en SIG se denomina modelado cartográfico y fue ampliado notablemente a través de las técnicas de evaluación multicriterio y el modelado prospectivo. La superposición raster con coincidencia espacio-temporal permite generar hipótesis basadas en la causalidad. En sistema vectorial es equivalente al análisis de la relación entre dos columnas de la tabla de atributos, lo cual desde un punto de vista estadístico lleva al concepto de correlación que se hace operativo con una medida precisa de la relación. La combinación de mayor número de variables permite la aplicación de procedimientos de análisis multivariado.

La "síntesis" espacial, desde un punto de vista cartográfico, corresponde a un resultado combinado de los diferentes aspectos espaciales generados en la investigación y a través de la cual puede contarse con elementos que brinden aspectos de la estructura socioespacial del área de estudio. La síntesis es una tarea geográfica por excelencia y generalmente aparece en resultados parciales mensurables y resultados finales con la respuesta espacial más completa y basada en la combinación de los conceptos centrales del análisis espacial. Los resultados presentados en la Figura $N^{\circ} 15$ y Figura $N^{\circ} 17$ corresponden a síntesis de la investigación.

El análisis conceptual, metodológico y aplicativo presentado en estas páginas muestra la vigente y fecunda relación que se produce entre la Geografía y la Cartografía, actualmente potenciada por las tecnologías de la información geográfica. El estudio de la dimensión espacial de la sociedad encuentra excelentes posibilidades para analizar, comprender y poder aplicar los resultados de la investigación científica tendientes a apoyar todo proceso de toma de decisiones orientado a mejorar la relación de la sociedad con su entorno.

\section{Referencias bibliográficas}

BUNGE, M. Emergencia y convergencia. Barcelona: Gedisa, 2003.

BUZAI, G.D. (director). Atlas digital de Luján. Formato CD. Luján: Universidad Nacional de Luján, 2001. 
BUZAI, G.D. (director). Atlas digital de la cuenca del río Luján - Sociodemográfico. Formato CD. Luján: Universidad Nacional de Luján, 2002.

BUZAI, G.D. Neogeografía y sociedad de la información geográfica. Una nueva etapa en la historia de la Geografía. Boletín del Colegio de Geógrafos del Perú, 2014, № 1, p. 1-12.

BUZAI, G.D. Geografía y Sistemas de Información Geográfica, 50 años. Boletín Red GESIG, 2015a, $N^{\circ} 1$, p. 2-3

BUZAI, G.D. Conceptos fundamentales del análisis espacial que sustentan la investigación científifica basada en geotecnologías. En: FUENZALIDA, M.; BUZAI, G.D.; MORENO JIMÉNEZ, A. y GARCÍA DE LEÓN, A. Geografía, Geotecnología y Análisis Espacial: Tendencias, métodos y aplicaciones. Santiago de Chile: Universidad Alberto Hurtado. 2015b, p. 56-72.

BUZAI, G.D. La Geografía como ciencia espacial. Bases conceptuales de la investigación astronómica vigentes en la Geografía Cuantitativa. Revista Universitaria de Geografía, 2016, Vol. 25, № 1 , p. 11-30.

BUZAI, G.D. y BAXENDALE, C.A. Análisis Socioespacial con Sistemas de Información Geográfica (Tomo 1). Buenos Aires: Lugar Editorial, 2011.

BUZAI, G.D. y BAXENDALE, C.A. Regionalización sociohabitacional de los partidos de la cuenca del río Luján (Provincia de Buenos Aires, Argentina). Anuario de la División Geografía 2016-2017. Luján: Universidad Nacional de Luján, 2017.

BUZAI, G.D.; BAXENDALE, C.A.; HUMACATA, L. y PRINCIPI, N. Sistemas de Información Geográfica. Cartografía temática y análisis espacial. Buenos Aires: Lugar Editorial, 2016.

BUZAI, G.D.; LANZELOTTI, S.L.; ACUÑA SUÁREZ, G.; BAXENDALE, C.A.; HUMACATA, L. y PRINCIPI, N. Análisis espacial y evaluación de zonas de potencial conflictos ambientales, productivos y patrimoniales ante la expansión urbana en la cuenca del río Luján (Provincia de Buenos Aires, Argentina). Perspectiva preliminar. Anuario de la División Geografía. Luján: Departamento de Ciencias Sociales, Universidad Nacional de Luján, 2015.

BUZAI, G.D. y PRINCIPI, N. Identificación de áreas de potencial conflicto entre usos del suelo en la cuenca del río Luján (Argentina). Revista Geográfica de América Central, 2017, № 59, p. 91-124.

BUZAI, G.D. y ROBINSON, D. Geographical Information Systems (GIS) in Latin America, 1987-2010: A Preliminary Overview. Journal of Latin American Geography, 2010, Vol. 9, N³, p. 9-31.

DACEY, M. Linguistics aspects of maps and geographic information. Ontario Geography. 1970, N 5, p. 71-80.

DOBSON, J.E. Automated Geography. The Professional Geographer, 1983, Vol. 35, № 2, p. 135-143.

GARCÍA, R. Sistemas Complejos. Barcelona: Gedisa, 2006. 
GONZÁLEZ, J.I. Tendencias cartográficas. Revista de Geografía Norte Grande, 1984, N 11, p. 3-16.

GONZÁLEZ, J.I. Las funciones de la Cartografía en los estudios geográficos. Revista de Geografía Norte Grande, 1986, №13, p. 21-27.

HAGGETT, P. Análisis Locacional en Geografía Humana. Barcelona: Gustavo Gili, 1977.

INSTITUTO NACIONAL DE ESTADÍSTICA Y CENSOS (INDEC). Censo Nacional de Población, Hogares y Viviendas 2010. Buenos Aires: INDEC, 2010.

LANZELOTTI, S.L. y BUZAI, G.D. Delimitación de la cuenca del río Luján, Provincia de Buenos Aires, Argentina. Informe técnico 01. PICT-2014-1388. Luján: Instituto de Investigaciones Geográficas, Universidad Nacional de Luján, 2015.

PASO VIOLA, L.F. Análisis cronológico documental de la evolución de la Cartografía en la alta cuenca del río Luján bajo la óptica de los procesos históricos globales y locales. Anuario de la División Geografía. Luján: Departamento de Ciencias Sociales, Universidad Nacional de Luján, 2013.

PASO VIOLA, L.F. Los Sistemas de Información Geográfica y la Cartografía Digital. Boletín Red GESIG,2016, N 4, p. 4-5

RABELLA, J.M. Mapa y SIG: medio siglo para un reencuentro... o del mito a un gran triunfo. Boletín Red GESIG, 2016, N 4, p. 6-8

RUSSELL, B. La perspectiva científica. Madrid: Sarpe. Madrid, 1985.

VON BERTALANFFY, L. Teoría General de los Sistemas. México: FCE, 1988. 
